\title{
El pasado en el presente. Métodos de elaboración cerámica vigentes en artefactos arqueologicos*
}

\author{
María Soledad Solórzano-Venegas**
}

RESUMEN

La parroquia rural La Pila, ubicada en el Cantón Montecristi, provincia de Manabí, alberga en su INTERIOR ALFARERos QUe A PARTIR de LA dÉCADA de loS AÑos 70 DEL SIGLO XX SE DEDICARON A LA PRODUCCIÓN ARTESANAL DE CERÁMICA CON DISEÑOS ARQUEOLÓGICOS COMO UNA ALTERNATIVA ECONÓMICA LOCAL. SE REGISTRó EN

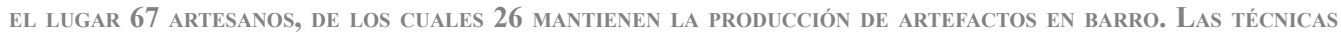
DE MANUfACTURA IDENTIFICADAS FUERON: MOLDE, MODELAdO SIMPLE, MODELAdO CON MOLDE Y MODELADO CON tabletas gruesas similar a un aCordelado. Se describe también el proceso de Quema en hornos a Cielo ABIERTO (DE LEÑA), DE TEMPERATURAS CONTROLADAS (LADRILLO) Y EL PROCESO DE ENVEJECIMIENTO DE LA CERÁMICA PARA QUE SEAN CONSIDERADAS COMO ORIGINALES.

Palabras Clave: la Pila - Alfareros - Reproducciones arqueológicas - Cerámica.

\section{Abstract}

In La Pila rural parish, located in Canton Montecristi in the province of Manabi, potters have been WORKING SINCE THE 70'S DEVOTED TO THE PRODUCTION OF HANDMADE CERAMICS WITH ARCHAEOLOGICAL DESIGNS AS A LOCAL ALTERNATIVE ECONOMIC ACTIVITY. 67 CRAFTSMEN, OF WHICH 26 PRESENTLY CONTINUE PRODUCTION OF

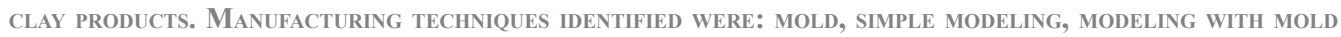
AND MODELING WITH THICK TABLETS SIMILAR TO AN ACORDELADO. WE ALSO DESCRIBE BURNING PROCESSES IN WOODFIRED OVENS, CONTROLLED- TEMPERATURE BRICK OVENS AND THE AGING PROCESS OF CERAMICS SO THAT THEY MIGHT BE CONSIDERED AS ORIGINAL.

Keywords: La Pila - Potters - archaeological reproductions - POTtery.

* El trabajo original del que surge este documento es la Investigación Arqueológica, Antropológica y Etnohistórica en la Parroquia Rural La Pila, Provincia de Manabí, auspiciado por el Instituto Nacional de Patrimonio Cultural Regional 4 y dirigido por la autora.

** Doctora en Arqueología \& Territorio. Correo electrónico: mssolorzano@hotmail.com 


\section{Introducción}

Oon la aparición de la alfarería, el ser humano descubrió la manera de controlar la transformación de los elementos naturales y así optimización su aprovechamiento. En este sentido, la arcilla, de ser un material plástico y quebradizo, pudo ser convertida en un producto duro y resistente, gracias a las transformaciones físicas (depuración, adhesión de agua y otros materiales, secado, amasado) y químicas (variaciones térmicas de temperatura) que ésta experimenta a través de la actividad alfarera (Childe, 1936).

La cerámica, que se convierte en el producto final de este proceso, mantiene atributos de resistencia y durabilidad notables frente a diversas condiciones ambientales y climáticas, de ahí que ésta sea uno de los elementos más recurrentes, utilizados para interpretar un sitio arqueológico (Orton, Tyers y Vince 1997).

La parroquia rural La Pila, posee una vasta riqueza arqueológica evidenciada a través de sus bienes culturales patrimoniales muebles e inmuebles. En base a la caracterización de la cerámica hallada en su territorio, tomando como base el trabajo de Estrada (1962), se ha definido ocupaciones vinculadas, principalmente, a los grupos Manteño (800 -1533 d.C) y Guangala (200 - 800 d.C.) (Solórzano, 2011).

Desde la década de los 70 del siglo $\mathrm{XX}$, haciendo uso de restos de cultura material arqueológica (moldes particularmente) se inició el proceso de producción de réplicas de artefactos arqueológicos en Manabí, en los sectores de Pila, Jama y Pedernales, principalmente.

La técnica manejada por los alfareros es empírica y a pesar de no tener un asidero teórico posee un alto nivel de perfección para dar los acabados a los artefactos con diseños arqueológicos.

En el presente trabajo se exponen las principales técnicas de producción cerámica en barro, que hoy en día se realizan en la Pila para la elaboración de artefactos arqueológicos.

\section{Metodología de investigación}

El inicio de la investigación etnográfica de la cerámica y sus procesos de producción empezó a mediados del siglo XIX, durante los trabajos de campo para la documentación de culturas no occidentales, retomado de manera más direccionada principalmente por las escuelas postprocesuales, consolidándose plenamente a finales del XX e inicios del XXI (Stark, 2003).

Para finales del siglo XX, los trabajos etnoarqueológicos presentan análisis de información que giran alrededor "de los modos de fabricación, la organización del trabajo y de los alfareros, el tipo de materias primas y de sus áreas de aprovisionamiento" (Durc, 1996: 20).

De acuerdo a González Rubial (2003) las etnografías en las que estudian la cerámica se pueden agrupar en dos grandes tipos:

Análisis de procesos tecnológicos

- Cadenas operativas de selección de materia prima

- Forma de montaje.

- Modelado

- Cocción.

- Simbología (rituales, cosmología).

- Aspectos sociales de la fabricación (género, marginación, identidad de los alfareros).

Análisis de distribución y consumo

- Estudio de mercados.

- Factores de selección de las vasijas

- Ciclos de la vida de la cerámica. 
En el presente documento se abordará el primer enfoque, bajo la consideración de que la etnoarqueología, al tomar evidencias del presente para interpretar a los grupos humanos pasado, puede ser considerada por sí misma como una metodología de obtención de información (Politis, 2002; Rubio 1998)

\section{Métodos de recolección de información}

La producción de artefactos ornamentales que involucra la transformación de barro o arcillas se da en tres tipos de materia prima en la Pila:

- El barro para réplicas arqueológicas. Comenzó en la década de los setentas del siglo XX, la mayoría de artesanos conocen su forma de preparación y manejo, a pesar de que no todos trabajan en él.

- El yeso. Si bien es un material cuyo trabajo debió iniciar junto al del barro en La Pila, para la extracción de moldes de piezas arqueológicas y posterior reproducción, en los últimos años se ha renovado, con la incorporación de cuadros y fruteros, entre otros elementos ornamentales.

- La barbotina. Se comenzó a trabajar con esta técnica a partir del segundo lustro del siglo XX, elaborando objetos un tanto más estilizados que con las otras materias primas, tales como floreros, objetos decorativos con diseños ornitomorfos -garzas, patos- de mamíferos (caballos, perros), alcancías, etc.

Durante el trabajo de campo se identificó un total de 67 talleres de producción cerámica destinados a los tres tipos de materia prima previamente descritos, en 26 de estos se dedican a trabajar réplicas arqueológicas ${ }^{1}$.

Una constante en los talleres es que estaban dirigidos o representados por hombres, las mujeres trabajan dando soporte, como personal auxiliar.

A todos los artesanos se les aplicaron encuestas direccionadas al trabajo de elaboración cerámica y los resultados iniciales llevaron a conocer que cualquiera que sea la materia prima a la que su taller se dedique, todos tienen conocimiento del trabajo en barro.

En lo referente a la recuperación in situ de los procesos de elaboración de cerámica observación directa- se dio énfasis al trabajo de cuatro alfareros (Víctor Bailón, Genaro López, Raúl López, María Piloso -Esposa de Jofre Velez).

Se debe resaltar que no todos los talleres cuentan con hornos para la quema de los objetos, principalmente en lo que a barro se refiere. Aproximadamente entre el 50 y $60 \%$ de los artesanos deben acudir a otros para poder quemar sus productos. Se observó de manera directa este proceso en el taller de los señores Genaro y Raúl López quienes tienen un horno de ladrillo². Estos artesanos, junto con Erasmo y Efraín López informaron sobre el proceso de quema en hornos de leña

La información sobre el envejecimiento de la cerámica fue posible documentar en base al trabajo con una sola persona, Richar Santana Mesone, uno de los pocos informantes que quiso compartir este conocimiento con el equipo de investigación.

De manera adicional, la familia Quijije Lucas y particularmente el Sr. Leonardo Quijije, quien en la actualidad tiene un taller dedicado a la producción de artefactos en barbotina pero que durante muchos años trabajó en barro, colaboró en el complemento de información recuperada en campo.

1 El trabajo de campo para la recolección de información se lo llevó a cabo entre mayo y julio de 2011.

2 Los talleres de los artesanos generalmente se encuentran en sus viviendas, al igual que las áreas destinadas para la quema, estas últimas se encuentran a una distancia prudencial de la zona de habitación -dependiendo del horno fluctúa entre 5 y 20 metros.

3 No se pudo documentar de manera directa el proceso en los hornos a cielo abierto o de leña, que en la actualidad se la utiliza principalmente para quemar jarras o material grande de entre 60 y $110 \mathrm{~cm}$ de alto. 
El trabajo fue documentado a través de fotografías, grabaciones de voz y de video, para posteriormente ser procesados, algunas imágenes presentadas en este artículo son capturas de las grabaciones realizadas.

\section{Procesos de producción de la cerámica con diseños arqueológicos en La Pila}

A raíz de una gran sequía en la década de los 70 en la provincia de Manabí, para subsistir, los habitantes de La Pila se dedicaron a la elaboración de artefactos cerámicos de barro, empleando moldes de piezas arqueológicas auténticas que les permitía la producción de réplicas, llegando en algunos casos a venderlas como originales (Martillo, 2011).

La técnica utilizada para la elaboración de artefactos cerámicos prehispánicos alcanzó tales niveles de perfección desde su inicio, que incluso, a arqueólogos con años de experiencia y altamente familiarizados con el material recuperado de contextos en la zona, les lleva a dudar el momento de diferenciarlos de los originales ${ }^{4}$.

En la actualidad, a parte de la incorporación de nuevas materias primas para el trabajo, los objetos de barro que se producen y comercializan se han diversificado, inicialmente se elaboraban figurillas -antropomorfas, zoomorfas, mixtas-, ollas de diferentes tipos y diseños -recreación de cultura material adscrita a la filiación Manteño, Valdivia, Chorrera, Machalilla, entre otros-; hoy en día se han incorporado otros diseños, por ejemplo ollas grandes tipo cántaros que sirven como floreros, pilares, etc.

Se han identificado tres macro - procesos al interior de la producción cerámica: preparación de la materia prima; elaboración del objeto y quema (transformación físico - química por cambios térmicos).

\section{Preparación de la arcilla}

Se conoce por la literatura científica que los procesos de preparación de la arcilla mantienen procedimientos muy similares en diferentes ecosistemas y continentes, con variaciones que se sustentan en el tipo de materia prima, volumen y productos terminados.

Los alfareros entrevistados coinciden en su totalidad que empezaron con la producción de cerámica en base a experimentación, aprendieron viendo cómo se elaboraba la cerámica ${ }^{5}$.

En la actualidad, los 26 talleres que trabajan con barro son abastecidos por un proveedor principal que trae la materia prima desde el cantón Jipijapa, de los "páramos" localizados en los sectores conocidos como La Naranja (40 kilómetros al suroeste de La Pila) y Noboa (aproximadamente 70 kilómetros al suroeste). En invierno el acceso a la "mina" es bastante complejo por lo agreste que se torna la vía debido a las lluvias, de acuerdo a lo que Leonardo Quijije explicó.

El barro de estos sectores posee la consistencia adecuada para la elaboración de objetos, a lo sumo necesita una depuración de macro impurezas lo que facilita el proceso de preparación: "El barro llega seco, luego se le coloca agua para "ponerlo a podrir", y empezar con el amasado en donde poco a poco se van retirando las impurezas, se deja así por lo menos tres días, para que esté "bien podrido y ablande", hasta que quede como una masa compacta y se procede a retirar las impurezas faltantes" (entrevista Víctor Bailón, mayo de 2011 en: Solórzano, 2011).

4 De manera anecdótica cuenta el Señor Richar Santana Mesone, que en una visita realizada al antiguo Museo del Banco Central, encontró unas piezas arqueológicas cuya autenticidad estaba siendo debatida, reconociendo entre ellas algunas de las realizadas por sus vecinos.

5 En la primera década de este siglo ingresa la Cooperativa Maquita Cushunchic en el sector. La fundación capacitó a los miembros de la comunidad en proceso de tecnificación del trabajo con barro, análisis de costo - beneficio para poder calcular los precios de venta, entre otros, de manera adicional se incorpora el trabajo con la barbotina mediante el acceso a créditos para el equipamiento para desarrollar su producción. 
Una vez limpia la masa y sólo en caso de ser necesario se le agrega arena de río (anti plástico). Se amasa el producto y se lo golpea buscando la consistencia ideal, "si está muy blandurito" no se puede trabajar. Se procede a formar bloques rectangulares de aproximadamente $40 \mathrm{~cm}$. por 30 $\mathrm{cm}$. y $8 \mathrm{~cm}$. de .espesor, que son pegados en la pared, dejándolos de un día para el otro. "Se sabe que ya está, cuando por lo menos uno de los bloques se desprende solo o fácilmente” (entrevista Genero y Raúl López en: Solórzano, 2011.

Los bloques son retirados de la pared y se los almacena para volver a amasarlos y darles la forma requerida, mediante el método de manufactura que escoja. En caso de que la masa se seca demasiado, se le adiciona el agua.

\section{Elaboración de los objetos}

En La Pila se identificaron dos técnicas de manufactura: Moldeado y Modelado. El modelado puede ser por cordel, simple o con la ayuda de moldes, con soporte de la técnica de paleteado.

\section{Modelado por tabletas, tipo cordel (acordelado)}

En base a los trabajos de María Pilos, quien colabora con su esposo en la producción de floreros tipo urnas y el de Jofre Velez quien elabora ollas tipo pondos, se documentó el trabajo de artefactos cuya técnica posibilita entender el proceso la creación de objetos arqueológicos de cuerpo elíptico.

El trabajo para la elaboración de estos elementos recuerda al acordelado, variando de este el tamaño de la tableta o cordel, siendo muy posible que en el periodo prehispánico haya podido ser utilizado este procedimiento.

Los objetos se los realiza por segmentos de manera separada: base y parte baja del cuerpo; y, cuello y borde.

El primer paso para elaborar cualquiera de las partes es hacer unas tabletas rectangulares y planas de barro, amasando y aplastando la arcilla contra el piso hasta conseguir un elemento de $40 \mathrm{~cm}$. de largo por $15 \mathrm{~cm}$. de ancho, con un espesor aproximado de entre 4 y 5 $\mathrm{cm}$., recortando los excedentes. El tamaño y número de las tabletas depende del objeto del que serán parte.

En el piso es colocado en un elemento circular del diámetro que se desee conseguir, a manera de soporte para empezar a "subir" la vasija poco a poco. Se coloca la arcilla en forma circular que tendrá la función de base sobre este elemento que fungirá de falso torno. Alrededor de la base se inician a colocar las placas rectangulares pegándolas con arcilla y agua.

Se va "cerrando" - sellando las zonas faltantes- también con la ayuda de arcilla y si es necesario de agua. Una vez que se termina el sellado de las placas, con el soporte de un artefacto de madera plano se dan ligeros golpes en la parte exterior mientras se sostiene el barro con la mano en el interior, esta tarea tiene la finalidad de homogenizar la pasta que se ha creado (paleteado). El proceso continúa hasta obtener la altura deseada, la cual es medida con una guía pre-establecida.

La siguiente parte del objeto se trabaja igual que la primera, con dos diferencias importantes: No se coloca la arcilla en la base, sin embargo si se inicia el proceso de levantamiento con la guía circular pre-definida que tendrá el tamaño que permita que se unan ambas. Las tabletas son más delgadas (entre 7 y $10 \mathrm{~cm}$. de ancho) aunque mantienen el largo y el espesor. Aparentemente el ancho de las tabletas ayuda a darle la forma al cuello. Al final se le elabora el borde, modelando la arcilla.

Una vez listas ambas partes, con los motivos que se hayan decidido colocar (apliques, acabados especiales) se las deja secar un tiempo prudencial hasta que la parte inferior pueda soportar a la superior.

La Señora Piloso señala que ella junto a su esposo se demoran dos días en preparar una docena y media de artefactos de este tipo. 
Previo a ser sometidas a fuego a las vasijas se las deja secar en sombra 3 días, para luego colocarlos al aire libre por dos días, al quinto se los coloca al sol, para finalmente meterlos en el horno: "Si hace sol las cosas tiemplan y secan más rápido, pero si está el clima frio demora" (Guido Bailón, en el taller del Sr. Víctor Bailón en: Solórzano, 2011).

La preparación de estas urnas y de la cerámica en barro en general, puede ser en piso con baldosa moderna, como es el caso de la Sra. Piloso, o en talleres con pisos de cualquier otro material, por ejemplo tierra, como es el caso del Sr. Erasmo López.

En el taller del Sr. López, en un espacio comprendido de $15 \mathrm{~m}^{2}$ se pudieron observar desniveles y elevaciones que quedan como testigos del proceso de producción cerámica en el piso.

El área donde se realiza el levantamiento de las piezas ligeramente más irregular que donde se ponen a secar y el lugar donde se les da el acabado y el pulimento.

Las vasijas de este tipo en la actualidad son quemadas en hornos de leña.

\section{Producción de cerámica modelada}

Se inicia el proceso separando la arcilla en bolas a las que se les da el tamaño basados en el del elemento que se desea elaborar, teniendo como principio la identificación de su forma y función.

En el caso de las representaciones zoomorfas de tamaño pequeño, la bola de arcilla es hueca de un grosor de la pared que le permita al alfarero retirar con objetos corto-punzantes o planos los excesos, dando forma al diseño que se desea conseguir, por ejemplo tortugas, mamíferos, aves, etc.

En el caso de los elementos compactos se mantiene el procedimiento de extracción y colocación de material quizás con menos delicadeza porque al ser una masa compacta no hay mayor problema con perforaciones -que son fácilmente subsanables en cualquier caso-, manteniéndose el control detallado en los acabados.

Está técnica también es utilizada para la producción de figurines eróticos y para la elaboración de figurines antropomorfos huecos, ayudándose con moldes, como se describe en la siguiente parte.

\section{Producción cerámica con moldes}

Se identifica dos formas de utilizar los moldes para la producción de artefactos.

El primero consiste en la obtención de improntas de las formas que se desea elaborar. Se identificaron dos tipos de moldes, los de origen arqueológico prehispánicos cuya materia prima es principalmente arcilla tipo terracota, y moldes modernos elaborados con yeso o barro, que en la mayoría de los casos son obtenidos de piezas originales o replicas con diseños pre-establecidos.

Se coloca la arcilla ya preparada en los moldes pegándola mediante pulso para grabar la forma.

Los objetos de tamaño pequeño generalmente necesitan de una sola impronta, sin embargo, en caso de artefactos grandes el procedimiento se va haciendo por partes, para posteriormente unirlos con ayuda de arcilla y agua retirando las impurezas.

La segunda forma de usar moldes se relaciona con la construcción de artefactos de diferentes formas para que la arcilla la adopte, similar a la descrita en el párrafo anterior.

Esta técnica se documentó para la realización de figurines antropomorfos, y también puede ser aplicada para la obtención de ollas globulares o elípticas, como ya se ha mencionado.

Se elaboran dos bolas de arcilla de tamaño similar aplanándolas con rodillo, con un objeto circular hueco se extrae la forma requerida por duplicado. Se colocan las placas circulares en un objeto globular para que obtenga la forma cóncava, una vez toman la forma deseada se pegan las dos tapas y se sellan con arcilla y agua. Se retiran los residuos. 
Cuando se ha formado un solo elemento se procede a golpearlo con un artefacto plano, hasta que las divisiones desaparecen y se obtiene la consistencia deseada. Se realiza un orificio central a manera de desfogue y con ayuda de golpes cada vez más delicados se obtiene la forma final requerida.

Haciendo uso de la técnica de paleteado, con cortes y adherencia de barro con ayuda de los dedos u objetos corto punzantes se obtiene el diseño deseado.

En la actualidad el trabajo con molde no solo se lo realiza para piezas arqueológicas, o de barro, también se lo utiliza en la elaboración de objetos modernos, como fruteros, frutas, alcancías de animales, apliques de adornos, entre otros, cuya materia prima no es el barro sino la barbotina.

\section{Tratamiento de superficie}

Una vez terminada (s) la (s) figura(s) se las deja unos días al aire libre para que estén bien secas y se procede a pulirlas, en el caso de que los diseños de los objetos los requieran.

El pulimento de las piezas de barro se realiza con piedras de diferente tamaño. Se inicia con piedras grandes, que son más fuertes y abarcan una mayor superficie. Para dar los terminados y llegar a los lugares más difíciles se utiliza rocas más pequeñas. Para el proceso se trabaja con cantos de ríos de diferente tipo y cuarzos, de acuerdo a lo que señalaron los informantes no hay un lugar específico de aprovisionamiento, simplemente cuando encuentran los idóneos son colectados y guardados.

El pulimento pre-cocción permite obtener un color negro en las piezas, sin embargo si la filiación cultural requiere otro color y para obtener diferentes tonalidades se hace uso de pinturas químicas que se consigue en los locales comerciales (ferreterías), a diferencia de épocas prehispánicas cuando se utilizaban tintes naturales.

\section{Quema}

Una vez que los artefactos se encuentran en estado de cuero seco se inicia el proceso de transformación físico - químico de la arcilla, en base a cambios térmicos, sometiéndolos a altas temperaturas.

Deben transcurrir por lo menos dos días después del pulido para poder entrar en este proceso.

En la Pila se pudo observar dos tipos de horno para la quema de cerámica con motivos arqueológicos, los hornos de leña a cielo abierto y los hornos de ladrillo.

\section{Horno de leña}

La leña debe estar suficientemente seca para que alcance una combustión total y facilite la quema homogénea de los artefactos, evitando que se partan o resquebrajen.

La madera para la leña proviene de diferentes tipos de árboles principalmente del muyuyo (Cordialutea), barbasco (Lonchocarpusnicou) y licuando, todos de la zona.

En el centro del área destinada para la quema se coloca una vasija parada, alrededor de ésta se ubican las otras en la misma posición, generalmente los artefactos deben tender al mismo tamaño.

La leña (tiras grandes de madera natural con un diámetro de entre 5 y $15 \mathrm{~cm}$ de diámetros y entre 1 y $1.5 \mathrm{~m}$ de largo) se va colocando de manera perpendicular y entre cruzada teniendo como guía las vasijas para que el fuego se distribuya de forma homogénea, acto seguido se enciende la leña con ayuda de papel y algún combustible para que inicie el proceso de combustión (gasolina o diésel).

Cuando las ollas ya están rojas de un lado se las va girando con ayuda de un instrumento largo de madera verde, para que no entre en el proceso de combustión.

Las ollas están paradas para que "la lengüetada le pegue" (llamarada grande). Durante la quema se van girando las vasijas y se las vas recostando sobre uno de los lados quedando en posición horizontal al final del proceso. 
Una vez terminado el proceso de combustión total de la leña y cuando se determina que las piezas están listas, se deja enfriar poco a poco y son retiradas del lugar al día siguiente.

Es necesaria mucha habilidad en esta actividad, para no quemarse y que los artefactos no se partan. Este proceso se aplica para objetos grandes y pequeños, la diferencia está en el tamaño de la hoguera. Cuando las piezas se fisuran o fracturan son resanadas con 'pegatubo' (goma blanca) para darles un acabado homogéneo ${ }^{6}$.

\section{Horno de ladrillo}

Con ladrillo se elaboran cajas cuadradas que en la parte baja posee una especie de loza que tienen dos desfogues, uno en cada extremo para que ingrese el aire y la temperatura se distribuya de forma equitativa.

Se llena todo el horno con piezas de distinto tamaño, en la parte baja se sitúan los más pesados, luego se colocan los más ligeros. Una vez que se llena el horno se cierra con ladrillo y barro para que se mantenga la temperatura estable. El barro que se utiliza es el mismo con el que se elaboran los objetos de cerámica.

Cuando está completamente sellado se procede a encender el mechero, que se encuentra en la parte baja de la caja y funciona con gas butano. Se lo deja encendido por un lapso aproximado de 5 horas, alcanzando temperaturas de aproximadamente $850^{\circ} \mathrm{C}$.

Las piezas se mantienen en el horno hasta el día siguiente. Luego de un proceso de enfriamiento lento, que tarda unas 12 horas se procede a romper el barro.

La arcilla utilizada para sellar el horno no puede ser reutilizada y es desechada.

\section{Proceso de envejecimiento}

Una vez quemadas las piezas y listas para la comercialización, algunas personas piden un tratamiento extra para que se asemeje más a las figuras precolombinas. Este método se llama envejecido. El señor Richar Santana Mesone explicó este proceso

El primer paso consiste en tomar un pedazo de cerámica auténtica que es lijada hasta obtener un polvillo; el producto se mezcla con diluyente, tiza blanca y pegamento de tubo (goma blanca). Con la mezcla obtenida se cubre la totalidad del artefacto para poder darle un empaste a manera de impermeabilizante.

Se escogen diferentes colores de pinturas modernas, compradas en la ferretería, considerando la cultura que se trata de imitar. Se mezclan los pigmentos con pegamento hasta obtener las tonalidades deseadas. Una vez listo se coloca el nuevo empaste con color requerido en el objeto cuando el primero ya está seco.

El siguiente paso es pulir la superficie con piedras de río o cuarzo liso para que tome brillo y no queden de un tono muy mate.

Finalmente se dan los acabados en los que se imita distintas condiciones de cocción y proceso de desgaste por el transcurso del tiempo que estuvieron enterrados. Con pigmentos negros se empieza a poner manchas en varios sitios para semejar partes quemadas durante la quema y que no se consiguen en los hornos de ladrillo. Después, con la ayuda de una brocha se lanza gotas finas de tintura negra para generar lo que llaman "el hongo negro de la tierra", que se forma sobre la pieza al estar enterrada durante varios cientos de años.

Como paso final se hace un último empaste que se realiza con "tierra de muerto", nombre que se le da a la tierra recogida de las distintas excavaciones donde se encontraron artefactos precolombinos, se la mezcla con pegamento y se pasa por toda la pieza.

6 En piezas que presentan fractura durante el proceso de cocción se puede observar un núcleo negro. 


\section{Conclusiones}

El presente trabajo expone procesos de producción cerámica contemporáneos de artefactos arqueológicos prehispánicos, partiendo del hecho de que tanto en el presente como en el pasado la cadena de producción responde a un condicionamiento social y ambiental.

En la Pila no se cuenta con una escuela donde se enseñe el proceso de producción cerámica, la mayoría de los alfareros han sido autodidactas o han adquirido el conocimiento siendo aprendices de otros talleres. El trabajo en barro para realizar réplicas arqueológicas fue espontáneo, surgió de la necesidad de obtención de recursos económicos en un periodo de austeridad y se convirtió en un modo de vida.

La producción alfarera en la Pila inicia con el trabajo en barro, sin embargo, por motivos de oferta y demanda, mediados por la tecnificación producto de la inserción de ONG's, se han incorporado nuevas materias primas.

La recopilación de información sobre la labor de los alfareros de La Pila, permitió identificar como cada uno de ellos pone su marca en la producción cerámica, y que forman parte de una cadena productiva con altos niveles de especialización, sintetizada en los siguientes puntos:

- El abastecimiento de materia prima se lo realiza por personas foráneas, comerciantes especializados que llegan de entre 40 y $70 \mathrm{~km}$ al suroeste (La Naranja y Noboa) de la Pila. A pesar de la distancia y el hecho de que durante el periodo invernal es difícil el acceso para la "mina", este material es muy apetecido por los habitantes del lugar, generando redes de contacto extra-locales.

- Los alfareros manejan todo el proceso de producción cerámica, desde la preparación del barro hasta la quema, en algunos casos llegan a la comercialización unitaria.

No obstante no todos trabajan en el mismo tipo de materia prima, incluso en los objetos de barro existe una diferenciación por filiación cultural o formas, unos se dedican a realizar figuras eróticas antropomorfas y zoomorfas, otros figurillas con diseños zoomorfos, hay quienes trabajan con tradiciones chorreras, o valdivias, gungala y otros en representaciones modernas.

Cada uno de los talleres tiene su marca personal, sea por el material con el que trabajan o el tipo de diseños que realizan, fortaleciéndose la especialización del trabajo y el respeto por el espacio del otro.

- La quema de los artefactos se la realiza en hornos especializados para cada producto. Si bien es cierto los alfareros de La Pila conocen sobre el proceso, no todos cuentan con hornos propios.

Los hornos de ladrillo en la actualidad se utilizan principalmente para objetos pequeños y de mediano tamaño, mientras que aún se conserva los hornos en leña para los objetos de gran tamaño. Con la barbotina se han introducido hornos industriales metálicos.

- Las redes intra-grupales se observan cuando alfareros que no poseen hornos propios alquilan a los que si los poseen, además de que cuándo el barro se les termina y tienen pedidos a la vista, entre ellos éste se presta o comercializa. Estas actividades se la realiza entre los miembros de la comunidad más afines, no se pudo documentar si existe una suerte de intercambio no monetario, más si las preferencias por aproximaciones. 
Del trabajo realizado en La Pila se desprenden símiles para entender el proceso de producción de cerámica arqueológica en los siguientes niveles:

- La arcilla procedente de Naranja y Noboa, en el pasado como en el presente, debió ser muy apetecida por los alfareros. Futuras investigaciones regionales de la zona podrían incorporar análisis especializados de los artefactos prehispánicos de la zona y compararlos con las fuentes de esta área.

- Las transformaciones físicas de la materia prima (barro) se las realiza mediante técnicas mecánicas, los acabados que se obtienen en este proceso se reflejan de manera clara en la forma del artefacto.

Existen objetos cerámicos al interior de los contextos arqueológicos, producidos en masa o no, que presentan ciertas deformidades tales como abultamientos o hundimientos, estos claramente son producto de una transformación física poco adecuada, en base del tipo de deformidad se puede conocer si es producto del amasado de la arcilla, un paleteado deficitario, o asociado a que el elemento no estuvo lo suficientemente seco previa a la transformación físico - química de la arcilla. Los defectos pueden ser producto de uno o varios factores combinados y claros para el ojo experto cuando empieza a buscarlos.

- Para los hornos a cielo abierto se hace uso de leña obtenida de árboles locales como el muyuyo, barbasco y liquando. Estas especies pudieron también ser utilizados desde el periodo prehispánico.

- En un mismo conglomerado social pueden coexistir diferentes tipos de hornos, lo que se vincula con el tipo de objetos que se van a introducir en estos (tamaño y cantidad) y el poder adquisitivo de los usuarios, ambos relativamente vinculados.

Se debe destacar que no necesariamente un ambiente de cocción a cielo abierto va a presentar en la cerámica núcleos grises o negros, ni en uno de cocción controlada se presentaran homogéneos, mucho depende del lugar donde fue colocada la pieza en el horno. En el caso de los hornos de leña, hay un dato adicional a ser considerado, si la pieza estuvo lo suficientemente seca o mantuvo algún grado de humedad, lo que se traducirá en los niveles de ahumado no intencional que tenga el objeto.

- La técnica de manufactura es de suma importancia el momento de analizar los artefactos prehispánicos y ser tomada con mucho cuidado, en tanto es claro que en un mismo objeto se pueden aplicar varios métodos de elaboración, por ejemplo las bases pueden ser modeladas, los cuerpos trabajados por cordel simple o con ayuda de paleteado, también haciendo uso de moldes y los bordes son terminados con modelado.

La coexistencia de varias técnicas de manufactura en un mismo objeto puede ser una constante, exceptuando de alguna manera los elementos que son producidos en masa con moldes pre-establecidos como se observa en el trabajo de yeso y barbotina.

- $\quad$ El alfarero va dando su toque a los artefactos, principalmente en el caso de los cavados de superficie (modelados, pulimentos) es un poco más visible. Aunque no se ha llegado a realizar el proceso de experimentación en relación al tema, es posible que en algunas piezas se pueda conocer si el alfarero es diestro, surdo, u otros atributos personales que pudieron quedar plasmados en el objeto.

El proceso de envejecimiento de los objetos arqueológicos, lleva a que la producción de la cerámica de La Pila haya dado verdaderos dolores de cabeza a los arqueólogos. El conocimiento 
sobre el uso de los elementos para dar este acabado puede que se pierda, aun cuando la producción de objetos con barro se mantenga, en tanto poco a poco han sido incorporadas nuevas materias primas y la normativa referente al tráfico ilícito se ha endurecido para la preservación de los bienes pertenecientes al patrimonio cultural del Ecuador.

La cadena de producción de objetos cerámicos de corte arqueológico, si bien es un conocimiento moderno, ha logrado reproducir piezas ancestrales a la perfección, por lo tanto las técnicas empleadas para su elaboración deben ser entendidas como parte del patrimonio inmaterial (no declarado) que tiene el Ecuador.

\section{Bibliografía}

Childe, V. 1936, Los orígenes de la civilización, Fondo de Cultura Económica, $21^{\circ}$ Reimpresión 1996, México.

Durc, I. 1996, "De la etnografía hacia la arqueología aprotes de entrevistas con ceramistas de Anchash (Perú) para caracterización de la cerámica prehispánica", en: Bulletin de l'Institut Français d'Études Andines, 25 (1), pp. 17-41.

Estrada, E. 1962, Arqueología de Manabí Central, Publicaciones del Museo Víctor Emilio Estrada, Guayaquil, Ecuador.

González-Ruibal, A. 2003, La Experiencia del Otro. Una introducción a la etnoarqueología. Madrid, Akal, España.

Martillo, J. 2011, La Pila: Memorias de barro. Recuperado el 20 de septiembre de 2015, de La Revista El Universo: http://www.larevista.ec/comunidad/cuerpo-y-alma/en-la-pila-memorias-de-barro.

Orton C., Tyers P., Vince A. 1997, La cerámica en Arqueología, Crítica Grijalbo Mondadori, Barcelona España.

Politis, G. 2002, Acerca de la etnoarqueolgía en América del Sur. Horizontes Antropológicos, ano 8, n. 18, pp. 61-91.

Rubio, I. 1998, La Etnoarqueología: Una disciplina nueva en la docencia universitaria y en la investigación españolas. CuPAUAM 251, pp. 9-33.

Stark, M. 2003, Current Issues in Ceramic Ethnoarchaeology. Journal of Archaeological Research, Vol. 11, No. 3, pp.193 - 242.

Solórzano M. 2011, Consultoría Investigación Etnohistorica, Arqueológica y Antropológica de la Parroquia Rural La Pila, Cantón Montecristi, Manabí, Informe Presentado al Instituto Nacional de Patrimonio Cultural, Quito (Informe inédito). 